\title{
Home sweet home cage
}

\author{
Why handle a mouse if your experiment can be done from the comfort of its home cage?
}

\author{
Ellen P. Neff
}

W hen a researcher says it's time to start a trial, lab animals don't have much say in the matter. Testing windows are usually set by a human's schedule, whether the subjects are hungry or satiated, tired or rested, bored or busy. But what if the animals had a choice?

"Choice is fundamentally about control," says Jamie Ahloy Dallaire, a postdoctoral researcher in Joseph Garner's lab at Stanford. Most animals, humans included, will choose what they feel is best for them, he says, whether that's engaging with a form of enrichment or avoiding a source of stress.

Much attention has been paid to standardizing variables across labs and testing protocols to tease out and control for the gene-environment interactions that are thought to contribute to the irreproducibility of preclinical work. But handling animals introduces stress that they can't avoid. For example, Jeffrey Mogil's lab at McGill University has documented stress-induced analgesia in mice handled by male experimenters; Jane Hurst in Liverpool has found that the traditional approach-picking mice up by the tail-is a contributing factor to anxiety that can impede their subsequent performance on different tests. To complicate matters further, rodents aren't usually active when experimenters want them to be. Working with animals one-by-one can also be time consuming for the humans involved.

An alternative is to just leave the animals at home. Passive video recording and radio-frequency identification devices (RFIDs) have let researchers keep track of what lab mice are up to for some time, but more active and complex tasks that require engagement from the animals are making their way to the home cage too.

\section{Tasks to translation}

Lynn Raymond at the University of British Columbia studies Huntington's Disease, a neurodegenerative disease characterized by a decline in motor function and cognitive abilities. When she decided to add a behavioral component to her mouse work, she chose to do so in the home cage. It has three main advantages, she says: continuous data and lots of it; testing task performance without introducing handling stress; and

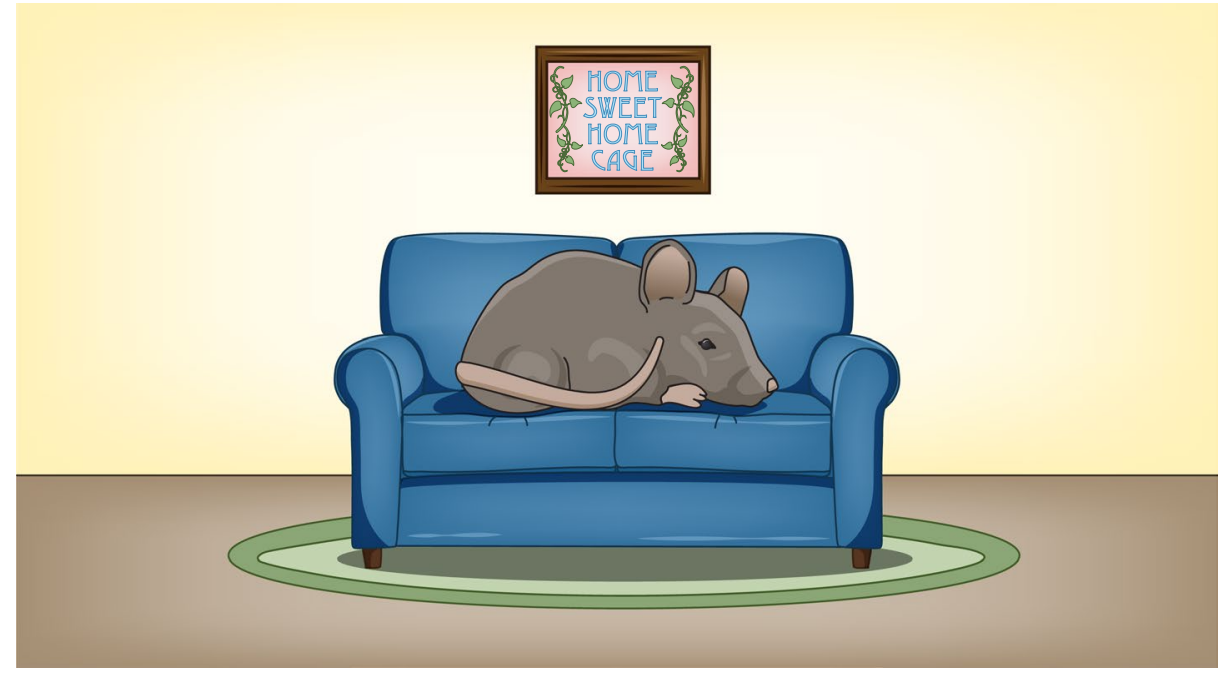

Staying put: When tasks can be performed from the home cage, the animals get more say in when to engage. Or relax. Credit: M. Spence / Springer Nature

tracking animals over time and alongside their littermates.

Working with her husband, Timothy Murphy, and his lab, Raymond and her graduate student Cameron Woodard recently adapted a skilled motor task-lever pulling-for the home cage ${ }^{1}$. The team built and attached a small Plexiglas chamber to the side of a home cage. Inside the chamber, there's a lever that RFID-tagged animals learn to manipulate to receive a water reward.

Such motor tasks usually involve removing the animals from their cage and obliging them to complete trials during a restricted window; the lever is available 24/7. That means Raymond and her colleagues can track how long it takes for an animal to learn the intended task-and adapt if the parameters change-as well as how they physically perform the intended motion. "We have all the information about the force that they're using and where they hold the lever. Is it steady? Is it jiggly? Do they pull it way back first and let it drift forwards again? Things like that, which are really pretty sensitive measures of things that we measure in the clinic in our patients," she says.

The home cage has given Raymond glimpses into declines that she says would have been harder to detect with discrete trials. At two months old, transgenic YAC128 mice, designed to model Huntington's Disease, couldn't keep up with changes to the task parameters as well as their wild-type littermates; at six months, the way they pulled the lever became irregular too. Because the animals could choose when to engage with the lever, the team also observed changes in their circadian rhythms.

Such observations are particularly relevant for her human patients, she says: cognitive decline usually precedes motor difficulties, and they'll often develop sleep disturbances as the disease progresses. "We can now use this as an outcome measure for treatments," she says.

Delivering those treatments could one day be done automatically too-her collaborators are working on an automated drinking system that can deliver potential therapies with the animals' water. How much each mouse consumes could be closely tracked, without needing to gavage them.

\section{Automating (pre)clinical care}

Ahloy Dallaire has also been developing an automated drinking system, but to study and assess pain. A human in pain will self-administer an analgesic; animals will too, if given the option he says. But studying that is laborious. He wants to up the 


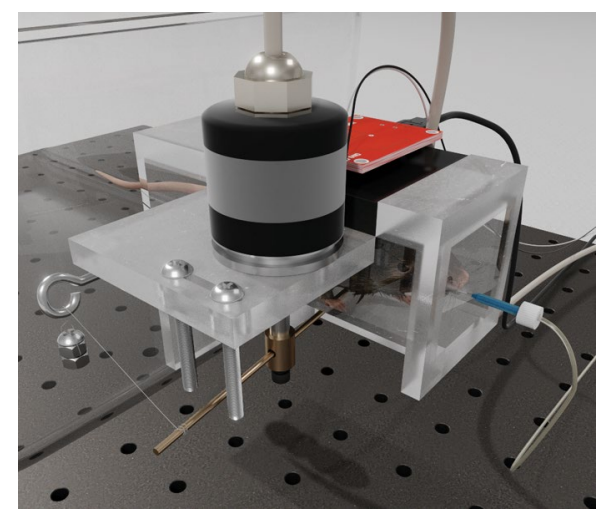

Pull! 3D-printed chambers can be customized to accommodate specific tasks, like lever pulling. Credit: C. Woodard, Raymond Lab

throughput and provide high-quality data "down to the drop."

The device he and the Garner lab are building is attached to the home cage, detects via RFID which mouse has come to the tap, and records exactly how much water they've each consumed. In preliminary data from their pilot device, they've found that mice are quite capable of responding to competing cues. Mice are group-housed and each is assigned a colored light that appears in a different position around a drinker port in the home cage. A given mouse can only get a drink when its respective light is on, so it must learn to ignore the cues that are specific to its cage mates.

Some mice get it more quickly than others, he says, but it's not a hands-on training process. "It's just setting the schedule and letting the mice do their thing." Lights come on in increasingly complex patterns as the mice learn which position \& color is theirs.

The lab would like to study mice as a model for how humans self-medicate, but automated drug delivery also holds promise for veterinary care, Ahloy Dallaire says. If a study involves a surgery, for example, the animals could medicate themselves with an analgesic via the drinking system as needed, rather than having to wait for a veterinarian or technician to make their rounds.

The standard for pain assessment in the clinic is self-reporting. "You're asking somebody, how bad does this feel and then they tell you," he says. "We would love to do that in mice but we can't, because we don't speak their language." Besides just delivering an analgesic, an automated system in the home cage could also be a novel way to assess pain.

In hospitals, patients can get a painkiller at the push of a button. Studies have correlated painkiller consumption with

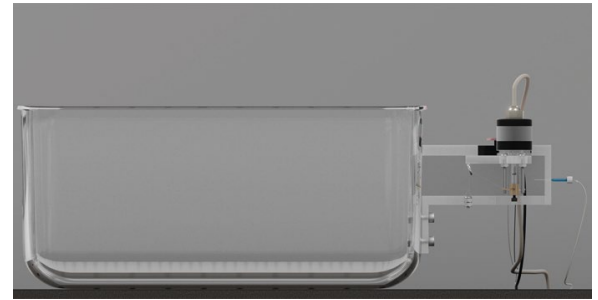

Easy attachment: Extending the home cage. Credit: C. Woodard, Raymond Lab

self-reporting, he says. Eventually the system will lock them out, but how much patients click in the interim and how quickly they react when the drug becomes available again can reflect of the severity of their pain. With the drinking system, Ahloy Dallaire and his colleagues hope to eventually develop a mouse version of pain self-reporting. Automated, and from the comfort of the animal's home cage.

\section{Neuroscience at home}

And there are more than just automated levers and lixits in the works to engage animals in their home cages. "I think there's a surprising amount that we wouldn't think at first glance could be done inside the home cage voluntarily but in the end it turns out can be done," Ahloy Dallaire says. Brain imaging is a notable example. "I absolutely would not have thought that could be possible, then somebody came up with it and it looked like it worked," he says.

In 2013, David Tank's lab at Princeton developed a system that could train rats to voluntarily position their heads under a twophoton microscope $e^{2}$. There's a similar proofof-concept for mice ${ }^{3}$.

In British Columbia, Murphy primarily studies stroke, and he wants to be able to follow mouse models over time and see how different interventions affect their brains. "I think having a home cage is an ideal model because it allows us to really follow the course of the disease model," he says. In addition to building levers for behavioral tasks with Raymond's lab, he's developed an automated system that can train mice to image their own brains under a wide-field microscope.

Imaging may seem like a daunting task to automate, but it came down to combining the right technology with existing protocols, he says. The animal care facility at the University of British Columbia had protocols for all the pieces he needed in place, like for head-restraining animals for imaging and using water restriction as training motivation. In consultation with the facility's veterinarians, Murphy and his lab developed a home cage system that combines those protocols with designs for automating the processes.

Each mouse gets an RFID for tracking purposes. Those used for in vivo imaging are implanted with a fixation bar that will hold their heads still and an optical window through which the microscope can record images. Brain coordinates are established as well so that the recording system can align itself automatically to each individual mouse. Inexpensive Raspberry PI microcomputers keep track of what's going on, and staff can check in at any point through video feeds.

Whenever the mouse wants a drink, it learns to fix its head under the microscope. In their published paper, they report over 7000 imaging sessions, completed without direct human supervision and done on the animal's own time.

In addition to recording images of the mouse cortex, Murphy wants to

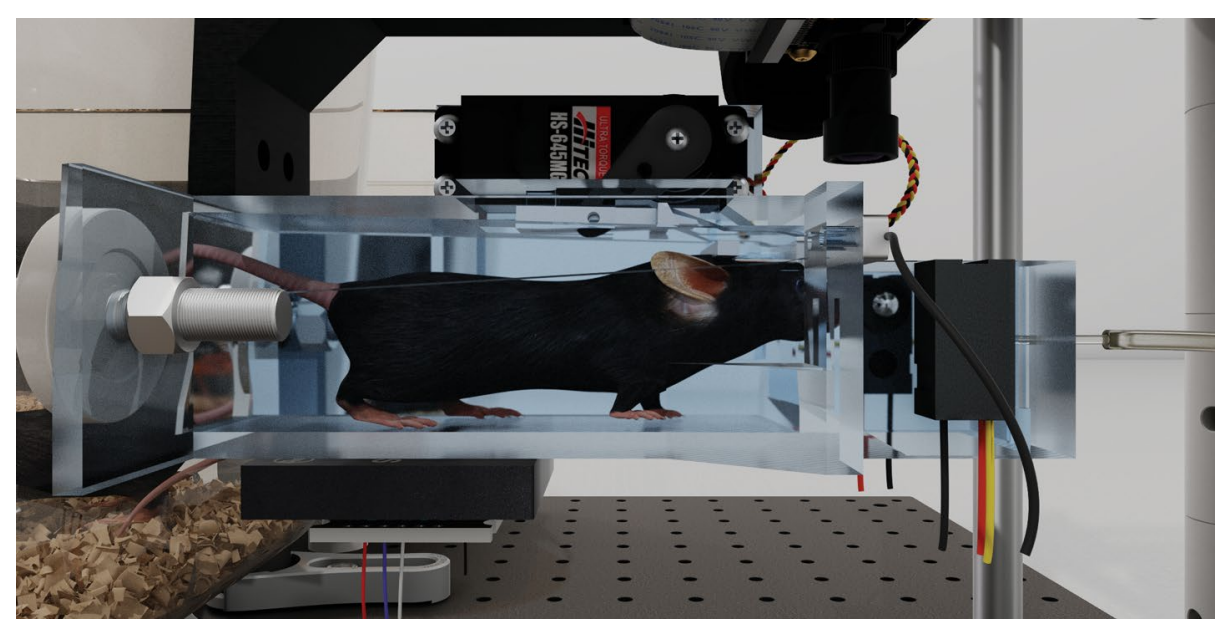

Fix yourself: Rendering of a self-head-restrained mouse. Credit: L. Bolaños \& T. Murphy 
expand the set in the future up to perform manipulations too, in a high-throughput manner that avoids handling the animals unnecessarily.

\section{Think like a mouse}

Andrea Benucci, a neuroscientist at the RIKEN Center for Brain Science in Japan, has watched with interest as other researchers developed automated head-fixing systems. But he saw limitations: the set ups were often designed for very specific goals. To him, the bottleneck was not recording, but training the animals. "That is the bottom line. You need to get mice trained."

He decided to separate the behavioral set-up from data acquisition, and his lab recently built a platform for self-head restraint that can be attached to two side-byside home cages, each housing an individual mouse $^{4}$. The platform is essentially a long corridor, with guiderails that steer a mouse, implanted with a head plate, towards a latching system where it learns to fix itself in place. It takes the animals about two weeks to get accustomed to self-fixing their heads, Benucci says. Once they're comfortable, researchers can add a task at the end of the tunnel and leave the mice to train themselves to complete it.

All these things can be done by a human, Benucci says, but manpower is costly.

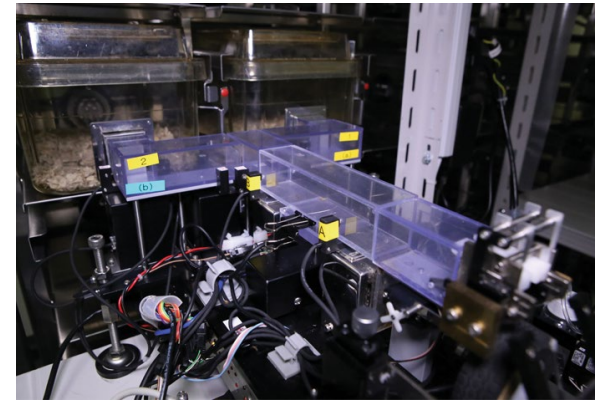

Custom ends: Benucci's corridor can accommodate different tasks. Credit: A. Benucci

And humans can be a bit of a co-factor themselves. "The way you fix the animal will change every time. The way you handle the animal will change. The human-toanimal interaction is a variable that's good to remove, from our point of view," he says.

Automating the training process can take a bit of thinking like a mouse. Benucci had to deal with escape artists: mice, with some creative yoga-like moves, that could manage to avoid his system's guiderails and squeeze out the end of the chamber. Others would take to sleeping in the corridor. Benucci's team had to make sure the tracking system, which detects where an animal is with infrared beams, wouldn't time out and lock a napping mouse inside the experiment platform.

Raymond recalls that their mice had a tendency to chew on the lever, instead of moving it with their paw. They now have to "nose-poke" against the end of the chamber to get their water while they simultaneously use their paw. A few of Ahloy Dallaire's mice weren't fond of their initial set-up either, he says. The way they had arranged the RFID reader meant the mice had to put most of their upper body into the drinking chamber to take a sip; a couple just wouldn't. A shallower chamber solved that reluctance.

"It's the kind of thing where you don't necessarily know until you try it what the hiccups are going to be," he says.

Researchers also have to keep their animals motivated when they have the option to engage with a task around the clock. Training with water rewards in the home cage means the mice always have water available, a potential welfare improvement over restricted training/testing protocols where the animals only receive water during the task itself. But could that make them lazy, particularly when faced with more complex tasks? When a little extra incentive might be needed, Murphy is considering mixing up the rewards. Sometimes the reward could be a sip of plain water, another time it could be a tasty

\section{Box 1 | Handling away from home}

Lab mice can't always stay home-some handling is evitable. The traditional method to pick up a mouse is by the base of its tail, supporting its weight with the opposite hand. But to a mouse, that's not ideal, says Jane Hurst, a researcher at the University of Liverpool. For the past few years, her lab has been comparing three handling approaches to picking up mice: by the tail, with a cupped hand, and by first shepherding them into a tunnel.

Hurst started using tunnels to handle wild mice that she was working with in the lab. When she started to use lab mice as "models" of wild rodents, she found them to be much more anxious than their wild brethren. "That's what led to our research to prove that actually handling them by the tail really does make those animals incredibly anxious," she says.

She's found that tunnel handled-mice, from a variety of strains, seem the least stressed and most engaged in subsequent behavioral tests ${ }^{5-7}$. Once picked up, the mice don't need to stay in the tunnel either-you can tip them backwards onto your hand, hold on to their tail to keep them put, or grab hold of their scruff as needed, she says. It's just that initial lifting action that seems to be so aversive.

"The difference in the behavior of the animals is astounding. I've never seen anything that has as big an effect as changing the handling method," Hurst says. "It's really, really surprising-people don't believe it until they've tried it for themselves."

She's encouraging others to consider the change, and some are starting to, she says. The animal facility at Liverpool piloted tunnel handling recently; once staff got used to the method, they could pick up the mice just as efficiently in tunnels as by the tail, she says. Tunnels have since been rolled out across the facility.

Other labs have since independently verified the use of tunnels, and Hurst has teamed up with the National Centre for the Replacement, Refinement \& Reduction of Animals in Research in the UK to produce training materials. Commercial animal supply vendors are starting to sell tunnels too.

“That's our recommendation, that you keep a tunnel in the cage so it's part of their normal environment," Hurst says. "I do think that that choice element is really very key." Although the animals don't actually get a say in the matter when it's time to leave the home cage, walking into a waiting tunnel on their own four paws seems to make a difference.

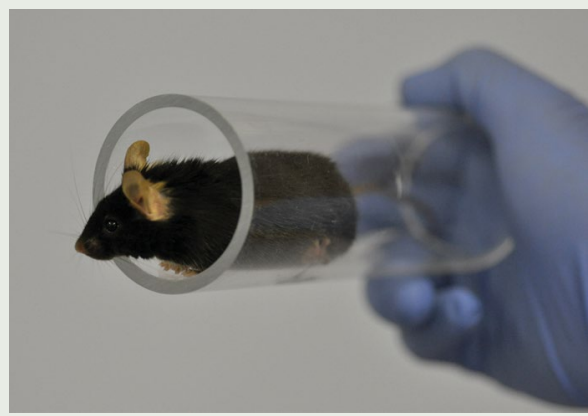

Tunnels: Lab mouse-approved. Credit: J. Hurst / J. Waters 


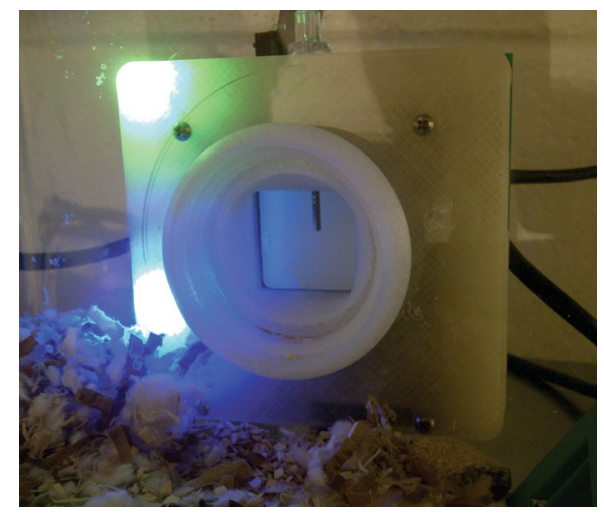

Lights on: Mouse-specific color cues. Credit: J. Ahloy Dallaire

strawberry milkshake. The mice won't know until they try.

\section{Off-the-shelf or do-it-yourself?}

Benucci's efforts to decouple training and data recording resulted in a modular system. The mice learn to fix their heads and perform whatever tasks the researcher intends, he explains. "Once the mouse is trained, the computer is going to tell you 'ok, this mouse is ready, put it under whatever system you want and just do the recording." That could be a video monitor or a wheel or lever for the animals to manipulate; a technician can then fix them under a widefield camera, or with electrical probes or optical fibers to manipulate the brain and record the results, Benucci says. "We're not committed to any specific set up."

Benucci's lab developed the platform in collaboration with O'Hara \& Co., a Japanese company that builds a variety of platforms and systems for behavioral tasks.

Other companies have home cages that can support automated tasks as well. Some are RFID-based. For example, Berlin-based PhenoSys offers a variety of extension chambers, like programmable touchscreens, that can be combined with a home cage. The IntelliCage, distributed by TSE Systems, consists of four operant corners where the animals can receive water rewards for performing a task. The company has recently been developing a series of behavioral chambers that can be added on as well, dubbed "PhenoWorld."

Noldus, headquartered in Wageningen, The Netherlands, has combined its video tracking system, EthoVision XT, with a home cage originally developed in collaboration with the Neuro-Bsik mouse phenotyping consortium. The home cage,
PhenoTyper, is modular-researchers can combine commercially available elements, like CognitionWall (an add-on developed by Amsterdam-based contract reserach organization Sylics) for learning and discrimination tasks, running wheels to measure activity, automated feeders or drinking bottles, or optogenetic \& calcium imaging hardware. EthoVision can also be used to program more customized hardware, if a researcher would like to add their own set-up.

But for labs that don't quite have the budget for commercial home cages, or that find themselves wanting to test something beyond current off-the-shelf capabilities, customization is getting easier.

It takes a level of interest and aptitude, but resources are there to help those who want to try their own hands at building automated systems. Murphy gets help from others at the university with engineering and physics backgrounds, and the neuroscientists in his lab have been training themselves in the basics of engineering and coding, with some help from YouTube. He makes his source code and 3d printer files freely available, and groups like OpenBehavior track and share details about open-source projects, including those for the home cage.

"But it's always a trade-off," says Murphy; commercial systems will have better documentation and support, but they might not be exactly what a researcher needs. Benucci has technical staff in his lab that he's happy to send to help train other groups interested in his system, but he thinks that having a plug-and-play product available in a catalogue, with the full-time customer support a commercial company can provide, is an advantage.

And there a lot of options out there. "You've got commercial teams, you've got academic teams," remarks Ahloy Dallaire, "I think there's a need for being able to integrate these different systems or different streams of information together because if you look at their capabilities, there are so many parameters."

\section{Hello homebodies}

The constant monitoring afforded by running experiments from the home cage is also enabling new observations about the mice themselves. The behaviors Benucci trains his animals to complete vary in complexity; some mice pick up even the hardest task with ease, while others will just give up. He's keeping track of the individuality of his mice, to see what might influence their performance and ability to learn.

As mice tend to be more active during dark phases, Murphy has noticed quite sporadic patterns of activity that seem centered on group dynamics and hierarchies among animals in the cage. Animals will do things in a certain order, and on their own time. Imposing artificial testing times might miss those details, he says.

But the home cage isn't the solution for everything. "There are going to be some types of data that you just can't collect in the home cage, that will just require that the animals be handled or that will require that they be put in an unfamiliar situation," says Ahloy Dallaire (see Box 1). But where possible, it's worth it, and the trend that's likely to continue.

"We're probably going to be doing all of our experiments this way in 25 years," Murphy predicts; tool building is still underway for the home cage, and it will be interesting to see what the next few years bring, he says.

"I'm a full professor and over 50 but I'm really excited about this project. Every day I come in and I want to work on it," he says, "It's something that I enjoy and feel challenged by."

In biomedical science, animals are generally intended to model humans, "and I think it can be important to give animals these meaningful choices and give them control over their environments," says Ahloy Dallaire, "because we, humans, are making meaningful choices all the time."

We have agency, he says, in the way we live our lives, the environment in which we do so, and "even as to whether or not we choose to become participants in health research. So I think giving themthe same kind of control in their housing environment and in the studies themselves can be a way of making them a better model of the humans they are meant to represent".

\section{Ellen P. Neff \\ Lab Animal, New York, New York, USA. \\ e-mail:Ellen.neff@us.nature.com}

Published online: 24 September 2018 https://doi.org/10.1038/s41684-018-0151-3

\footnotetext{
References

1. Woodard, C. L. et al. eNeuro 5, e0141-17.2017 (2017).

2. Scott, B. B., Brody, C. D. \& Tank, D. W. Neuron 80, 371-384 (2013)

3. Murphy, T. H. et al. Nat Commun 7, 11611 (2016)

4. Aoki, R., Tsubota, T., Goya, Y. \& Benucci, A. Nat Commun 8, 1196 (2017).

5. Hurst, J. L. \& West, R. S. Nat Methods 7, 825-826 (2010).

6. Gouveia, K. \& Hurst, J. L. PLoS One 8, e66401 (2013).

7. Gouveia, K. \& Hurst, J. L. Sci Rep 7, 44999 (2017).
} 